\title{
PRIMITIVE THERAPUTIC BIDS OF MEDICINAL PLANT ROHITAKA- TECOMELLA UNDULATA (SM.) SEEM.: A COMPREHENSIVE APPRAISAL BASED ON CLASSICAL AYURVEDA TEXTS
}

\section{Santosh Kumar Thakur*}

\section{Sumit Natthani}

\section{Mita Kotecha}

2-3PG Department of Dravyaguna Vigyana, National Institute of Ayurveda, Amer Road, Jaipur-302002, Rajasthan, India. ${ }^{*}$ Corresponding Author

2-3PG Department of Dravyaguna Vigyana, National Institute of Ayurveda, Amer Road, Jaipur-302002, Rajasthan, India.

2-3PG Department of Dravyaguna Vigyana, National Institute of Ayurveda, Amer Road, Jaipur-302002, Rajasthan, India.

ABSTRACT The nature made world is graduallyeyeing towards plant based natural system of medicine like Ayurveda. The novel healing approach of Ayurveda founded on evidence based fundamental approaches are gaining interest of the scientific communities globally. There are various interesting therapies are in the ocean of Ayurveda, still looking for scientific exploration in order to serve the people worldwide. The plant Rohitaka botanically identified as Tecomellaundulata Sm. which is commonly distributed in drier part of North-West and western India, is such an example. The present review aimed to analyze the ancient therapeutic uses of Rohitaka mentioned in classical texts of Ayurveda and systematically categorized them in term of contemporary practices. The review suggests that in spite of various published study there are still scope of exploration different novel use of Rohitaka mentioned in classical Ayurveda texts. The present reviews may perform an optimistic role for Researcher, Physician and Industries. It may also provide the future leads and scope for drug development and product marketing.

\section{KEYWORDS : Plants, Rohitaka, Hepatic, Tecomella, Traditional}

\section{INTRODUCTION}

Latest decade have professed remarkable growth in demand of plant based medicine and herbal product in International market. Contemporary data shows that medicinal plants accounts for nearly $70 \%$ by worth of the total raw material procured by Ayurvedic companies. The time honored Ayurveda science has gained its importance in the global arcade due to its unique healing approaches. The ancient fundamental base of Ayurveda for novel therapeutic applications of nature based medicine is now the point of attraction of the scientific communities. ${ }^{[1-6]}$

The plant Rohitaka isa small tree up to $8 \mathrm{~m}$ high with drooping, hairy branches; botanically identified as Tecomellaundulata Sm.which is commonly distributed in drier part of North-West and western India; extending from western arid/semi-arid parts of Gujarat, Rajasthan, Delhi, and Punjab to eastwards in parts of Uttarpradesh, Bihar along the river Yamuna ascending up to an altitude of $1200 \mathrm{~m} .^{[7-9]}$

Rohitaka is described in Atharvaveda as a plant used for YagyaKarma (ritual purpose). Similarly it's wood was used for making stick for UpanayanaSanskar(oath ceremony) of Kings.(Pai.sam-9/7/13).The wood of Rohitaka wasin used for lighting fire by rubbing.In Ayurveda Rohitaka is famous for treating liver related disorders. It isdescribed as a liver and spleen tonic and indicated in the case ofhepatomegaly. ${ }^{[10]}$ Various isolated chemical compounds of Rohitaka have also been documented. ${ }^{[1-12]}$ Classical text books of Ayurveda Nighantus(lexicons) are also widely prescribed this plant in the conditions like yakrutadaliudara, kamala, kumbha kamala, panaki etc. (disorders associated liver and spleen pathophysiology).

The present review aimed to analyse the ancient therapeutic uses of Rohitaka mentioned in classical texts of Ayurveda and systematically categorized them in term of contemporary proctises.

\section{POLEMICASPECTS OF ROHITAKA:}

The term Rohitaka is found in various classical texts of Ayurveda. The confusion arises when in CharakaSamhita $13^{\text {th }}$ Chapter of ChikitsaAdhyaya, RohitakaLata(climber or creeper) is mentioned. While in Nighantus(like ShodhalNighantu, KaidevaNighantu and Raja Nighantu)two varieties of Rohitaka i.e. Rakta and SwetaRohitaka are mentioned, which is also creates controversy about accurate and genuine source of both varieties of Rohitaka. But the identity of Rohitaka is clearly indicated in its descriptive names given classics, such as Dadimchhadaand Dadimpushpaka(leaves and flower are similar like-DadimaPunicagranatum L.). But due to its distribution only in western parts of India and non-availability to other parts practices of substitution with Aphanamixis polystachya (Wall.) R.Parkerand other species also creates controversy in therapeutic uses of original species. Similarly Parijata the name given by Bopadeva as synonyms of Rohitaka also a cause of controversy because Parijata. Parijata and Paribhadra appear to be synonyms and may be identified with species of Erythrina (Erythrina variegata L.). Nowadays maximum number of scholars are agreed Tecomellaundulata (Sm.) Seem. as the genuine botanical source of Rohitaka and it is also mentioned in Ayurvedic Pharmacopoeia of India (API). The name of Rohitakalata in CharakaSamhita, appears to indicate the use of thin hanging branches of Tecomellaundulata (Sm.) Seem. The other species which are available in markets of different parts of country are given in the table (Table-1), those are used due to non-availability of T.undulata everywhere and those are establishing as substitute and adulteration of genuine source. ${ }^{[13-14]}$

Table- 1. Different regional source of Rohitaka:

\begin{tabular}{|l|l|l|l|}
\hline $\begin{array}{l}\text { S. } \\
\text { No. }\end{array}$ & Latin name & Family & $\begin{array}{l}\text { Place where } \\
\text { used }\end{array}$ \\
\hline 1. & $\begin{array}{l}\text { Aphanamixixpolystachia } \\
\text { (Blatter) }\end{array}$ & Meliaceae & Bengal \\
\hline 2. & $\begin{array}{l}\text { Mabanigrescens Dalz and } \\
\text { Gibs }\end{array}$ & Ebenaceae & Mumbai \\
\hline 3. & $\begin{array}{l}\text { Ventilagomadraspatana } \\
\text { Gaertn. }\end{array}$ & Rhamnceae & Dang, Pavagadh \\
\hline 4. & Polygonum glabrum Willd. & Palygonaceae & $\begin{array}{l}\text { Mahabaleshwar, } \\
\text { Pavagadh }\end{array}$ \\
\hline 5. & Myristica attenuate Wall & Myristicaceae & North Kanara \\
\hline 6. & $\begin{array}{l}\text { Rhamnus wightii Wight \& } \\
\text { Arn. }\end{array}$ & Rhamnaceae & Nilgiris \\
\hline 7. & Tecomellaundulata & Bignoniaceae & $\begin{array}{l}\text { Rajputana, } \\
\text { Ratlam }\end{array}$ \\
\hline
\end{tabular}

GJRA - GLOBAL JOURNAL FOR RESEARCH ANALYSIS 17 
Table-2: Rasapanchaka(pharmacological traits) of Rohitaka:The basic fundamental properties of Rohitaka as mentioned in Ayurveda as bellow-

\begin{tabular}{|l|l|l|}
\hline Sl. No & Traits & Entities \\
\hline l. & Rasa (taste) & $\begin{array}{l}\text { Katu (pungent),Tikta (bitter), } \\
\text { Kashaya(astringent) }\end{array}$ \\
\hline 2. & Guna(virtue) & $\begin{array}{l}\text { Sara (movable), Snigdha } \\
\text { (unctuous) }\end{array}$ \\
\hline 3. & Veerya(potency) & Sheeta(cold) \\
\hline 4. & Vipaka(metabolism) & Katu(pungent) \\
\hline 5. & Prabhava & $\begin{array}{l}\text { Plihaghna(cure spleen } \\
\text { disorders) }\end{array}$ \\
\hline 6. & Dosh Karma (action) & Kaph Pitta Nashaka \\
\hline
\end{tabular}

Table-3. Sansthanik karma of Rohitaka:Specific system wise pharmacological actions of Rohitaka in Ayurveda:

\begin{tabular}{|l|l|}
\hline Doshakarma (action) & Kaphapittashamaka \\
\hline $\begin{array}{l}\text { Sansthanik karma } \\
\text { (external action) }\end{array}$ & $\begin{array}{l}\text { Chakshushya (eye } \\
\text { protective),Vranaropana(wound } \\
\text { healing) }\end{array}$ \\
\hline $\begin{array}{l}\text { Pachansansthan } \\
\text { (digestive system) }\end{array}$ & $\begin{array}{l}\text { Deepan (carminative),Pachan } \\
\text { (digestive),Anulomana } \\
\text { (laxative),Krimighna(anthelmintics) }\end{array}$ \\
\hline $\begin{array}{l}\text { Rasvahasansthan } \\
\text { (lymphatic system) }\end{array}$ & Hridya(cardioprotective) \\
\hline $\begin{array}{l}\text { Mutravahasansthan } \\
\text { (urinary system) }\end{array}$ & $\begin{array}{l}\text { Mutrasangrahaniya (regulator } \\
\text { urine),Kaph- ittajprameha } \\
\text { (diabetes) }\end{array}$ \\
\hline $\begin{array}{l}\text { Raktavahasansthan } \\
\text { (haematological } \\
\text { saytem) }\end{array}$ & $\begin{array}{l}\text { Plihasankochaka (spleen } \\
\text { ailment),Rakta-shodhaka(blood } \\
\text { purifier) }\end{array}$ \\
\hline $\begin{array}{l}\text { Prajanansansthan } \\
\text { (genial system) }\end{array}$ & $\begin{array}{l}\text { Yonishravrodhaka } \\
\text { (control abnormal discharge) }\end{array}$ \\
\hline Satmikarana(habitual) & $\begin{array}{l}\text { Lekhana (scrapping),Vishaghna } \\
\text { (antidote) }\end{array}$ \\
\hline
\end{tabular}

THERAPUTIC APPLICATIONS OF ROHITAKA IN DIFFERENT CLASSICAL TEXTS OF AYURVEDA: Rohitaka described under different multi-ingredients formulations (yoga) are listed in table (Table-4).

Table-4. Formulations (yoga) of Rohitaka mentioned in CharakaSamhita: ${ }^{[15]}$

\begin{tabular}{|l|l|l|l|l|}
\hline $\begin{array}{l}\text { S. } \\
\text { No. }\end{array}$ & Name of Yoga & Indications & $\begin{array}{l}\text { Dosage } \\
\text { form }\end{array}$ & Reference \\
\hline 2. & Rohitkadiyog & $\begin{array}{l}\text { Udarroga } \\
\text { (gastric disorders) }\end{array}$ & Decoction & $\begin{array}{l}\text { Ch.chi } \\
13 / 81-84\end{array}$ \\
\hline 3. & Rohitakghrita & $\begin{array}{l}\text { Udarroga } \\
\text { (gastric disorders) }\end{array}$ & Ghee & $\begin{array}{l}\text { Ch.chi } \\
\text { l3/83-85 }\end{array}$ \\
\hline 4. & Triphaladikwath & $\begin{array}{l}\text { Udarroga } \\
\text { (gastric disorders) }\end{array}$ & Decoction & $\begin{array}{l}\text { Ch.chi } \\
\text { l3/149 }\end{array}$ \\
\hline 5. & $\begin{array}{l}\text { UdumbaradiLe } \\
\text { ha }\end{array}$ & $\begin{array}{l}\text { Hridrog } \\
\text { (heart disease) }\end{array}$ & Avaleha & $\begin{array}{l}\text { Ch.chi } \\
26 / 98\end{array}$ \\
\hline 6. & Rohitakkalka & $\begin{array}{l}\text { Sweta-Pradar } \\
\text { (leucorrhoea) }\end{array}$ & Paste & $\begin{array}{l}\text { Ch.chi. } \\
30 / 116\end{array}$ \\
\hline 7. & $\begin{array}{l}\text { Kampilladi } \\
\text { Yoga }\end{array}$ & $\begin{array}{l}\text { Prameha } \\
\text { (diabetes) }\end{array}$ & Powder & $\begin{array}{l}\text { Ch.chi } \\
6 / 35\end{array}$ \\
\hline
\end{tabular}

Note: Ch.-CharakaSamhita, chi-Chikitsasthan

Table-5. Formulations (yoga) of Rohitaka mentioned in SushrutaSamhita: ${ }^{[16]}$

\begin{tabular}{|l|l|l|l|l|}
\hline $\begin{array}{l}\text { S. } \\
\text { no. }\end{array}$ & Name of Yoga & Indications & $\begin{array}{l}\text { Dosage } \\
\text { form }\end{array}$ & Reference \\
\hline l. & Bhallatkadimantha & $\begin{array}{l}\text { Mahakustha } \\
\text { (skin disease) }\end{array}$ & Mantha & $\begin{array}{l}\text { Su.chi. } \\
10 / 4\end{array}$ \\
\hline 2. & $\begin{array}{l}\text { Kutajkapitha } \\
\text { dikashaya }\end{array}$ & $\begin{array}{l}\text { Prameha } \\
\text { (diabetes) }\end{array}$ & Kwath & Su.chi.11/8 \\
\hline 3. & RasakriyaAnjan & $\begin{array}{l}\text { KachRoga } \\
\text { (eye disease) }\end{array}$ & Anjan & $\begin{array}{l}\text { Su.U. } \\
17 / 41\end{array}$ \\
\hline
\end{tabular}

Note:Su.-SushrutaSamhita, chi-Chikitsasthan, U-Uttar tontra

Table-6. Formulations (yoga) of Rohitaka mentioned in AstangaSamgraha: ${ }^{[17]}$

\begin{tabular}{|l|l|l|l|l|}
\hline $\begin{array}{l}\text { S. } \\
\text { No. }\end{array}$ & Name of Yoga & Indications & $\begin{array}{l}\text { Form of } \\
\text { drug }\end{array}$ & $\begin{array}{l}\text { Referenc } \\
\text { e }\end{array}$ \\
\hline 1. & RohitkyadiAvleha & $\begin{array}{l}\text { Hridrog } \\
\text { (heart disease) }\end{array}$ & Avaleha & $\begin{array}{l}\text { A.sam.c } \\
\text { hi. 8/33 }\end{array}$ \\
\hline 2. & $\begin{array}{l}\text { Salsaptaparnyadichur } \\
\text { na }\end{array}$ & $\begin{array}{l}\text { Prameha } \\
\text { (diabetes) }\end{array}$ & Powder & $\begin{array}{l}\text { A.sam.c } \\
\text { hi. 14/5 }\end{array}$ \\
\hline 3. & $\begin{array}{l}\text { Triphaladantyadighrit } \\
\text { a }\end{array}$ & $\begin{array}{l}\text { Udarroga } \\
\text { (gastric disorder) }\end{array}$ & Ghee & $\begin{array}{l}\text { A.sam.c } \\
\text { hi 17/12 }\end{array}$ \\
\hline 4. & Rohitakshatpalghrita & $\begin{array}{l}\text { Udarroga } \\
\text { (gastric disorder) }\end{array}$ & Ghee & $\begin{array}{l}\text { A.sam.c } \\
\text { hi 17/34 }\end{array}$ \\
\hline 5. & Rohitakghrita & $\begin{array}{l}\text { Udarroga } \\
\text { (gastric disorder) }\end{array}$ & Ghee & $\begin{array}{l}\text { A.sam.C } \\
\text { hi 17/35 }\end{array}$ \\
\hline 6. & Rohitkadi yoga & $\begin{array}{l}\text { Udarroga } \\
\text { (gastric disorder) }\end{array}$ & Hima & $\begin{array}{l}\text { A.sam.c } \\
\text { hi 17/36 }\end{array}$ \\
\hline 7. & Bhunimbadichurna & $\begin{array}{l}\text { Kustha } \\
\text { (skin disease) }\end{array}$ & Powder & $\begin{array}{l}\text { A.sam.C } \\
\text { hi 21/40 }\end{array}$ \\
\hline 8. & Rohitakjatakalka & $\begin{array}{l}\text { Pradar } \\
\text { (leucorrhoea) }\end{array}$ & Paste & $\begin{array}{l}\text { A.Sam.U } \\
\text {. 39/73 }\end{array}$ \\
\hline
\end{tabular}

Note: A.sam.-AstangaSamgraha, chi- Chikitsasthan, U-Uttar tantra

Table-7.Formulations (yoga) of Rohitaka mentioned inAstangaHridaya: ${ }^{[18]}$

\begin{tabular}{|l|l|l|l|l|}
\hline $\begin{array}{l}\text { S. } \\
\text { No. }\end{array}$ & Name of Yoga & Indications & $\begin{array}{l}\text { Forms of } \\
\text { drugs }\end{array}$ & Reference \\
\hline 1. & $\begin{array}{l}\text { Rohitka } \\
\text { dikwatha }\end{array}$ & $\begin{array}{l}\text { Hridrog } \\
\text { (heart disease) }\end{array}$ & Decoction & $\begin{array}{l}\text { A.Hri.chi. } \\
6 / 53\end{array}$ \\
\hline 2. & $\begin{array}{l}\text { Pramehanashak } \\
\text { yoga }\end{array}$ & $\begin{array}{l}\text { Prameha } \\
\text { (diabetes) }\end{array}$ & Powder & $\begin{array}{l}\text { A.Hri.chi. } \\
12 / 16\end{array}$ \\
\hline 3. & Rohitakghrita & $\begin{array}{l}\text { Udarroga } \\
\text { (gastric disorders) }\end{array}$ & Ghee & $\begin{array}{l}\text { A.Hri.Chi. } \\
15 / 91-94\end{array}$ \\
\hline
\end{tabular}

Note: A.Hri.-AstangaHridaya, chi- Chikitsasthan, U-Uttar tantra

Table-8. Formulations (yoga) of Rohitaka mentioned in KashyapaSamhita: :119] $^{10}$

\begin{tabular}{|l|l|l|l|l|}
\hline $\begin{array}{l}\text { S. } \\
\text { No. }\end{array}$ & $\begin{array}{l}\text { Name of } \\
\text { Yoga }\end{array}$ & Indications & $\begin{array}{l}\text { Dosage } \\
\text { form }\end{array}$ & Reference \\
\hline 1. & $\begin{array}{l}\text { Rohitk } \\
\text { adikwatha }\end{array}$ & $\begin{array}{l}\text { Pleehadoshara } \\
\text { manartha }\end{array}$ & Decoction & $\begin{array}{l}\text { Katutailakalpa/ } \\
10\end{array}$ \\
\hline 2. & $\begin{array}{l}\text { Parijat } \\
\text { kshara }\end{array}$ & $\begin{array}{l}\text { Pleehadoshasa } \\
\text { manartha }\end{array}$ & Kshara & $\begin{array}{l}\text { Katutailakalpa/ } \\
16.17\end{array}$ \\
\hline
\end{tabular}

Table-9. Formulations (yoga) of Rohitaka mentioned in SharnagadharaSamhita: ${ }^{[20]}$

\begin{tabular}{|l|l|l|l|}
\hline $\begin{array}{l}\text { S. } \\
\text { No }\end{array}$ & Name of Yoga & $\begin{array}{l}\text { Dosage } \\
\text { form }\end{array}$ & Reference \\
\hline l. & Pathyadikwath & Decoction & Sha.sam.ma.kh. 2/121 \\
\hline 2. & Rohitakarista & Arista & Sha.sam.ma.kh. 12/73-76 \\
\hline
\end{tabular}

Note: Sha. sam. ma. kh.- Sharangadhar Samhita Madhyam Khanda.

Table-10. Formulations (yoga) of Rohitaka mentioned in YogRatnakarSamhita: ${ }^{[21]}$

\begin{tabular}{|l|l|l|l|l|}
\hline $\begin{array}{l}\text { S. } \\
\text { No. }\end{array}$ & Name of Yoga & Indications & $\begin{array}{l}\text { Form of } \\
\text { drug }\end{array}$ & Reference \\
\hline 1. & $\begin{array}{l}\text { Kampilladi } \\
\text { churna }\end{array}$ & $\begin{array}{l}\text { Prameha } \\
\text { (diabetes) }\end{array}$ & Powder & Prameh chi. \\
\hline 2. & $\begin{array}{l}\text { Rohitakabhara } \\
\text { kalka }\end{array}$ & $\begin{array}{l}\text { Udarroga } \\
\text { (gastric disorders) }\end{array}$ & Paste & $\begin{array}{l}\text { Plihodarud } \\
\text { ar chi. }\end{array}$ \\
\hline 3. & $\begin{array}{l}\text { Maharo } \\
\text { hitakghrit }\end{array}$ & $\begin{array}{l}\text { Udarroga } \\
\text { (gastric disorders) }\end{array}$ & Ghee & Udar chi. \\
\hline 4. & $\begin{array}{l}\text { Rohita } \\
\text { kmoolkalk }\end{array}$ & $\begin{array}{l}\text { Pradar } \\
\text { (leucorrhoea) }\end{array}$ & Paste & Pradar chi. \\
\hline
\end{tabular}

Note: chi- Chikitsasthan. 
Table-11. Formulations (yoga) of Rohitaka mentioned in Chakradutta: ${ }^{[22]}$

\begin{tabular}{|l|l|l|l|l|}
\hline $\begin{array}{l}\text { S. } \\
\text { No. }\end{array}$ & Name of Yoga & Indications & $\begin{array}{l}\text { Dosage } \\
\text { form }\end{array}$ & Reference \\
\hline 1. & $\begin{array}{l}\text { Kampilladi } \\
\text { churna }\end{array}$ & $\begin{array}{l}\text { Prameha } \\
\text { (diabetes) }\end{array}$ & Powder & $\begin{array}{l}\text { Ch.da. } \\
37 / 46 / 20\end{array}$ \\
\hline 2. & $\begin{array}{l}\text { Rohitakabhaya } \\
\text { churna }\end{array}$ & $\begin{array}{l}\text { Udarroga } \\
\text { (gastric disorders) }\end{array}$ & Powder & $\begin{array}{l}\text { Ch.da. } \\
37 / 46\end{array}$ \\
\hline 3. & $\begin{array}{l}\text { Rohitakabhaya } \\
\text { kwath }\end{array}$ & $\begin{array}{l}\text { Plihayakrit chi. (liver } \\
\text { \& spleen ailment) }\end{array}$ & Decoction & Ch.da.38/4 \\
\hline 4. & $\begin{array}{l}\text { Rohitakharita } \\
\text { kiprayog }\end{array}$ & $\begin{array}{l}\text { Plihayakrit chi. } \\
\text { (liver \& spleen } \\
\text { ailment) }\end{array}$ & Powder & $\begin{array}{l}\text { Ch.da.38/1 } \\
3\end{array}$ \\
\hline 5. & Rohitakghrit & $\begin{array}{l}\text { Plihayakrit chi. } \\
\text { (liver \& spleen } \\
\text { ailment) }\end{array}$ & Ghee & $\begin{array}{l}\text { Ch.da.38/3 } \\
8-40\end{array}$ \\
\hline 6. & $\begin{array}{l}\text { Maharohitakg } \\
\text { hrit }\end{array}$ & $\begin{array}{l}\text { Plihayakrit chi. } \\
\text { (liver \&spleen } \\
\text { ailment) }\end{array}$ & Ghee & $\begin{array}{l}\text { Ch.da } \\
38 / 41-48 .\end{array}$ \\
\hline 7. & Rohitakkalk & $\begin{array}{l}\text { Pradar chi. } \\
\text { (spleen ailment) }\end{array}$ & Paste & $\begin{array}{l}\text { Ch.da- } \\
61 / 4 .\end{array}$ \\
\hline
\end{tabular}

Note: Ch. da-Chakra dutta.

Table-12.Formulations (yoga) of Rohitaka mentioned inVangasenaSamhita: ${ }^{[23]}$

\begin{tabular}{|l|l|l|l|l|}
\hline $\begin{array}{l}\text { S. } \\
\text { No. }\end{array}$ & Name of Yoga & Indications & $\begin{array}{l}\text { Dosage } \\
\text { form }\end{array}$ & Reference \\
\hline 1. & $\begin{array}{l}\text { Kampilladi } \\
\text { churna }\end{array}$ & Prameha(diabetes) & Powder & $\begin{array}{l}\text { Vang.se. } \\
38 / 52\end{array}$ \\
\hline 2. & $\begin{array}{l}\text { Rohitak } \\
\text { haritakikalk }\end{array}$ & $\begin{array}{l}\text { Udarroga } \\
\text { (gastric disorders) }\end{array}$ & Paste & $\begin{array}{l}\text { Vang.se. } \\
40 / 47\end{array}$ \\
\hline 3. & Rohitakghrit & $\begin{array}{l}\text { Udarroga } \\
\text { (gastric disorders) }\end{array}$ & Ghee & $\begin{array}{l}\text { Vang.se. } \\
40 / 79-82\end{array}$ \\
\hline 4. & Rohitakkalk & $\begin{array}{l}\text { Pradar } \\
\text { (leucorrhoea) }\end{array}$ & Paste & $\begin{array}{l}\text { Vang.se.69 } \\
/ 32\end{array}$ \\
\hline
\end{tabular}

Note: Vang.se.-VangasenaSamhita

Table-13. Formulations (yoga) of Rohitaka mentioned in GadaNigraha: ${ }^{[24]}$

\begin{tabular}{|l|l|l|l|l|}
\hline $\begin{array}{l}\text { S. } \\
\text { No. }\end{array}$ & Name of Yoga & Indications & $\begin{array}{l}\text { Dosage } \\
\text { form }\end{array}$ & Reference \\
\hline l. & Rohitakghrit & $\begin{array}{l}\text { Kustha(skin } \\
\text { disease) }\end{array}$ & Ghee & $\begin{array}{l}\text { Ga.ni.pra.k } \\
\text { ha.1/58-60 }\end{array}$ \\
\hline 2. & Rohitakghrit & $\begin{array}{l}\text { Udarroga(g } \\
\text { astric } \\
\text { disorders) }\end{array}$ & Ghee & $\begin{array}{l}\text { Ga.ni.pra.k } \\
\text { ha 1/176- } \\
\text { l82 }\end{array}$ \\
\hline 3. & Ayorajchurna & $\begin{array}{l}\text { Udarroga(g } \\
\text { astric } \\
\text { disorders) }\end{array}$ & Powder & $\begin{array}{l}\text { Ga.ni.pra.k } \\
\text { ha 3/411- } \\
421\end{array}$ \\
\hline 4. & Rohitakvatak & $\begin{array}{l}\text { Udarroga(g } \\
\text { astric } \\
\text { disorders) }\end{array}$ & Tablet & $\begin{array}{l}\text { Ga.ni.pra.k } \\
\text { ha 4/70 }\end{array}$ \\
\hline 5. & Rohitakavaleha & $\begin{array}{l}\text { Hridrog } \\
\text { (heart } \\
\text { disease) }\end{array}$ & Avleha & $\begin{array}{l}\text { Ga.ni.pra.k } \\
\text { ha 5/105- } \\
\text { 106 }\end{array}$ \\
\hline 6. & Rohitakasava & $\begin{array}{l}\text { Pradar(leuco } \\
\text { rrhoea) }\end{array}$ & Aasava & $\begin{array}{l}\text { Ga.ni.pra.k } \\
\text { ha 6/115- } \\
\text { 117 }\end{array}$ \\
\hline 7. & Rohitakasava & $\begin{array}{l}\text { Prameha(dia } \\
\text { betes) }\end{array}$ & Asava & $\begin{array}{l}\text { Ga.ni.pra.k } \\
\text { ha.1/58-60 }\end{array}$ \\
\hline 8. & Kampilladichurna & - & $\begin{array}{l}\text { Ga.ni.pra.k } \\
\text { ha 6/337- } \\
\text { 34l. }\end{array}$ \\
\hline 9. & Rohitakabhayapr \\
ayog & $\begin{array}{l}\text { Prameha(dia } \\
\text { betes) }\end{array}$ & Powder & $\begin{array}{l}\text { Ga.ni.dwi.k } \\
\text { h. 30/55 }\end{array}$ \\
\hline 10. & $\begin{array}{l}\text { Rohitakakrisnaprors) } \\
\text { ayog }\end{array}$ & $\begin{array}{l}\text { Ga.ni.dwi.k } \\
\text { h 2/32/66- } \\
68\end{array}$ \\
\hline
\end{tabular}

\begin{tabular}{|l|l|l|l|l|}
\hline 11. & $\begin{array}{l}\text { HaritakiRohitakya } \\
\text { divati }\end{array}$ & $\begin{array}{l}\text { Udarroga(g } \\
\text { astric } \\
\text { disorders) }\end{array}$ & Tablet & $\begin{array}{l}\text { Ga.ni.dwi.k } \\
\text { h 2/32/90 }\end{array}$ \\
\hline 12 & $\begin{array}{l}\text { Rohitakabharyapr } \\
\text { ayog }\end{array}$ & $\begin{array}{l}\text { Udarroga(g } \\
\text { astric } \\
\text { disorders) }\end{array}$ & Tablet & $\begin{array}{l}\text { Ga.ni.dwi.k } \\
\text { h 2/32/112 }\end{array}$ \\
\hline 13 & Rohitakghrit & $\begin{array}{l}\text { Udarroga(g } \\
\text { astric } \\
\text { disorders) }\end{array}$ & Ghee & $\begin{array}{l}\text { Ga.ni.dwi.k } \\
\text { h 2/132/134 }\end{array}$ \\
\hline
\end{tabular}

Note: Ga.ni.pra.kha-GadaNigrahaSamhitaPrathamKhanda, Dwi-dwitiya.

Table-14. Formulations (yoga) of Rohitaka mentioned inBhaishajyaratnavali: ${ }^{[25]}$

\begin{tabular}{|c|c|c|c|c|}
\hline \begin{tabular}{|l|} 
S. \\
No.
\end{tabular} & Name of Yoga & Indications & $\begin{array}{l}\text { Dosage } \\
\text { form }\end{array}$ & $\begin{array}{l}\text { Referenc } \\
\text { e }\end{array}$ \\
\hline \begin{tabular}{l|l}
1. \\
\end{tabular} & Nidigdhikakwath & \begin{tabular}{|l|}
$\begin{array}{l}\text { Kustha(skin } \\
\text { disease) }\end{array}$ \\
\end{tabular} & Ghrit & \begin{tabular}{|l|} 
Bhai.Ra. \\
5/44 \\
\end{tabular} \\
\hline 2. & Panchgavyaghrit & $\begin{array}{l}\text { Udarroga(g } \\
\text { astric } \\
\text { disorders) } \\
\end{array}$ & Ghrit & \begin{tabular}{|l} 
Bhai.Ra. \\
$25 / 40$
\end{tabular} \\
\hline 3. & Rohitakkalk & \begin{tabular}{|l|} 
Udarroga(g \\
astric \\
disorders) \\
\end{tabular} & Churna & $\begin{array}{l}\text { Bhai.Ra. } \\
\text { 40/26 }\end{array}$ \\
\hline 4. & Rohitabharyakwath & \begin{tabular}{|l|} 
Udarroga(g \\
astric \\
disorders)
\end{tabular} & vatak & \begin{tabular}{|l|} 
Bhai.Ra.4 \\
0/10-11
\end{tabular} \\
\hline 5. & RohitakGhrit(mahat) & \begin{tabular}{|l|}
$\begin{array}{l}\text { KaphajHrid } \\
\text { rog }\end{array}$ \\
\end{tabular} & Avleha & $\begin{array}{l}\text { Bhai.Ra.4 } \\
\text { 0/23-40 }\end{array}$ \\
\hline 6. & Rohitakarista & $\begin{array}{l}\text { Udarroga(g } \\
\text { astric } \\
\text { disorders) }\end{array}$ & Arista & \begin{tabular}{|l|} 
Bhai.Ra.4 \\
0/235-238
\end{tabular} \\
\hline 7. & RohitakPrayog & \begin{tabular}{|l|} 
Plihayakrit \\
chi. (liver \\
\&spleen \\
ailment) \\
\end{tabular} & churna & $\begin{array}{l}\text { Bhai.Ra. } \\
41 / 15\end{array}$ \\
\hline 8. & Rohitakvati & \begin{tabular}{|l|} 
Plihayakrit \\
chi. (liver \& \\
spleen \\
ailment) \\
\end{tabular} & Vati & \begin{tabular}{|l|} 
Bhai.Ra.4 \\
1/22-24
\end{tabular} \\
\hline 9. & PlihshardulRas & \begin{tabular}{|l|} 
Plihayakrit \\
chi. (liver \& \\
spleen \\
ailment)
\end{tabular} & Ras & \begin{tabular}{|l|} 
Bhai.Ra.4 \\
1/99-104
\end{tabular} \\
\hline 10. & RohitakLauh & \begin{tabular}{|l|} 
Plihayakrit \\
chi. (liver \& \\
spleen \\
ailment)
\end{tabular} & Vati & \begin{tabular}{|l|} 
Bhai.Ra.4 \\
1/117
\end{tabular} \\
\hline 11. & NyagrodhadyaGhrit & \begin{tabular}{|l|} 
Plihayakrit \\
chi. (liver \& \\
spleen \\
ailment)
\end{tabular} & Ghrit & $\begin{array}{l}\text { Bhai.Ra.6 } \\
\text { 6/93-99 }\end{array}$ \\
\hline
\end{tabular}

Note:Bhai. Ra.-Bhaishajyaratnavali.

\section{DISCUSSION:}

Ayurveda explains multi-dimensional therapeutics applications of Rohitaka (Table-3-13). While analysis such indications some interesting novel applications are also need to be brought for exploration. It is mentioned in classicsthat stem bark of Rohitakashould be kept in decoction of Haritaki or cow urine for a week and the extract is takento alleviateJaundice (Kamala), Gulma, Prameha, Piles(Arsha), Pleeha (spleenomegaly) and otherUdara (enlargement of abdomen) and worms. During the course of the treatment meat-soup of wild animals should be taken. (Ch.Chi.13/81-82 \&A.H.Chi. 15/91-92). Intake of cow's urine or water impregnated with powder of Rohitaka and Haritaki alleviates all types of abdominal enlargement (Udara) including Pleehodara (spleen enlargement), Prameha, Arsha, Krimi and 
Gulma. (V.M.-37/51). One suffering from Prameha(diabetes) caused by Kapha and Pitta should take powdered flowers of Kampillaka, Saptaparna, Shala, Vibhitaki, Rohitaka, KutajaandKapittha with honey. (Ch.Chi. 6/35). For bath and internal administration, Khadira, Aragvadha, Arjuna, Rohitka, Lodhra, Kutaja and Kapittha with honey should be taken, that may alleviatesKushtha (skin disorders) (Ch.Chi. 7/129). According to ChakraduttaRohitaka is drug of choice in liver disorders and Vrana. (C.D. 321 ). He also explainedInternal administration of paste of root of Rohitaka (RohitakaMool Kalka) is beneficial for Pandu (anaemia). (C.D. $30 / 116) .{ }^{[26-27]}$

Various studies have also established its multidimensional pharmacological properties like-Antibacterial, antifungal, antiviral, antifertility, hypoglycaemic, CNS depressant, anticancer, hepatoprotective, hypotensive, muscle relaxant, cardic depressant, diuretic, analgesic etc.. The researchers opined that thehepatoprotective activity of Rohitakapossibly may be a result of the presence of flavonoid compounds. ${ }^{[27-33]}$

In spite of various published study there are still scope of exploration different novel use of Rohitaka mentioned in classical Ayurveda texts. The present reviews may perform an optimistic role for Researcher, Physician and Industries. It may also provide the future leads and scope for drug developmentand product marketing.

\section{REFERENCES:}

[1] Thakur, S., Kotecha, M., Natthani, S., \& Mahajon, B. (2018). Pharmacognostical Standardization of Stem Bark of Rhododendron arboreum Sm.(Pullas). Journal of Advances in Medicine and Medical Research, 25(8), 1-11.https://doi.org/10.9734/JAMMR/2018/40391.

[2] Mahajon, B., \& Murthy, R. (2017). Traditional Herbal Remedies for Complications of Diabetes Mellitus. SciFed Journal of Herbal Medicine, 1(1).10.23959/sfjhm-1000004

[3] Mahajon, B. \& Murthy, A. R. (2018). Relevance of Shramaharamahakashaya (An anti-fatigue formulation) in sports medicine. Journal of Scientific and Innovative Research, 7(1), 15-17.

[4] Mahajon, B., \& Murthy, A. R. (2017). A comprehensive analysis on shrama (fatigue) in Ancient transecript of charakasamhita. Global Journal For Research Analysis, 6(6), 346-47.

[5] Bidhan, M., Rupashri, N., \&Remadevi, R. (2014). Ascientific review on dietetics in Ayurveda according to Prakriti (innate constitution). IAMJ, 2(6), 1031-1036.

[6] Mahajon, Bidhan \& M, Deepak \& B, Remashree\&Remadevi, R. (2015). Phytochemical analysis of ayurvedickashaaya residues to assess its reusability. 2. 1049-1059.

[7] Kirtikar K.R., Basu B D. (1987). Indian Medicinal Plants vol-3, International Book Distributors-Dehradun.pp.1838.

[8] Kirtikar K.R., Basu B D, (1987) Indian Medicinal Plants vol-3, International Book Distributors-Dehradun.pp. 1841

[9] Quality standards of Indian medicinal plants. (2005).vol 3, co-ordination and editing AK Gupta, NeerajTandon, Madhu Sharma, ICMR-New Delhi.pp.340341.

[10] VanwariLal Mishra, DrvayagunaHastamlaka, 1st edition, Jaipur: Publication Scheme; 1976.p.150-51

[11] Theodore Cook, Flora of Presidency Bombay, (1976)Vol-II, 2nd reprint edition, Mumbai: Shree Swarasati Press Limited.pp.402

[12] Ram PratapRastogi BN Mehrotra,(1993) Compendium of Indian Medicinal Plants, Vol-II, Re print edition, Delhi: Publication Information Directorate.pp.667.

[13] P.C. Sharma, M.B. Yelne, T.J.(2005) Dennis ( Eds.). Database on Medicinal Plants used in Ayurveda. Central Council for Ayurveda and Siddha. Vol.-6. New Delhi.pp.321

[14] Singh, T. B., \&Chunekar, K. C. (1972). Glossary of vegetable drugs in Brhattrayi. Varanasi: Chowkhamba Sanskrit Series Office. pp. 164-165.

[15] Agnivesh, LaxmidharDwivedi. (2016). Hindi commentator, CharakaSamhita, Part III, 2nd edition, Varanasi: Chowkhamba Krishna Das Academy.pp.268, 301, 449, 459, 859, 1007.

[16] Acharya Sushruta, Ambika Dutta Shastri Hindi commentator. SushrutaSamhita, Part-I and II, Reprint Edition, Varanasi: The Chowkhamba Sanskrit Samsthana; 2070BS.pp. 71,76.

[17] Acharya Bhagvata, P Shrinivas Rao. (2008) English commentator, AstangaSamgraha, Vol-II, lst Edition, Varanasi:Chowkhamba Krishna Das Academy.pp.394, 467, 499, 504.

[18] Acharya Bhagvata, HarisadashivShatri Hindi commentator AstangaHridaya, Vol-II, Reprint Edition, Varanasi: Chowkhamba Sanskrit Samsthana; 2069BS.p.625, 679,698.

[19] Acharya Kashyap, P.V. (2008) Tiwari English commentator KashyapSamhita, Reprint Edition, Varanasi:Chowkhamba Vishwabharati.pp.340-41.

[20] Acharya Sharangadhara, ShalijaShrivastava.(2015) Hindi commentator Sharangadharasamhita, Reprint Edition, Varanasi: Chowkhamba orientalia.pp.153, 253-54.

[21] IndrdevaTripathi, Daya Shankar Tripathi.(2013), Yogratnakara, 4th Edition, Varanasi: Chowkhamba Krishna Das Academy.pp.512, 555, 531, 832.

[22] Chakrapani, IndradevTripathi.(2015)hindi editor, Chakradutta, Reprint Edition, Varanasi: Chowkhamba Sanskrit Bhavana.pp.217-376
[23] Vangasen,PanditHarihar Prasad Tripathi, (2016). hindieditor,Vangasen Samhita,2nd edition, Varanasi: Chowkhamba Sanskrit series office.pp.143,152,182,191

[24] Shodhala, IndrdevaTripathi, Ganga sahayapandeyhindi editor Gadnigraha, Reprint Edition, New delhi; Chowkhamba publication ; 2069BS.p.28, 214, 245, 313, 368, 396, 672, 700, 704, 708, 712, 713.

[25] Govind das sen, SidhiNandan Mishra,(2015).Bhasajyaratnawali Hindi editor, Varanasi; ChowkhambaSurbharati prakashana.pp.128, 515, 747-766 1036.

[26] Mahajon, B., \&Remadevi, R. (2015). Folk medicine for YakritVikaara (Liver Disorder)-A Scientific Review. IAMJ, 3(6), 1828-1847.

[27] Mahajon, B., \&Nath, R. R. (2014). Remadevi. A Scientific Analysis of Single Drug Remedies in Chakradutta (A Convenient Ayurvedic Transcript). J Pharm Scilnnov, 3(6), 492-4.

[28] Rana, M. G., Katbamna, R. V., Dudhrejiya, A. V., \&Sheth, N. R. (2008). Hepatoprotection of Tecomellaundulata against experimentally induced liver injury in rats. Pharmacologyonline, 3, 674-682.

[29] D. VithyaEaswari, S. N. Suresh, Sagadevan.P, S. Rathish Kumar. Studies on Phytochemical Composition and Anticancer Potential of Methenolic Lea Extract of TecomellaUndulata. www.pharmaresearchlibrary. com/tag/tecomella-undulata/

[30] Soni P K, Mali P C. (2017). Evaluate Antifertility Effects Of TecomellaUndulata To Developan Oral Male Contraceptive. Indian Journal of Applied Research. 7 (7): 341-344

[31] Rajwant K. Kalia, Manoj K. Rai, Ramavtar Sharma, R. K. Bhatt. (2014) Understanding Tecomellaundulata: an endangered pharmaceutically important timber species of hot arid regions. Genet Resour Crop Evol. 61:1397-1421

[32] Ahmad F, Khan RA, Rasheed S. (1994) Preliminary screening of methanolic extracts of Celastruspaniculatus and Tecomellaundulata for analgesic and anti-inflammatory activities. J Ethnopharmacol. 42(3):193-8.

[33] Rohilla R, Garg M.(2014) Phytochemistry and pharmacology of Tecomellaundulata. Int J Green Pharm. 8:1-6. 